\title{
Aggregation kinetics of binary systems containing kaolinite and Pseudomonas putida induced by different 1:1 electrolytes: Specific ion effects
}

\author{
Zhaoxuan Yu ${ }^{1}$, Rui Tian ${ }^{\text {Corresp., }}{ }^{1}$, Dian Liu ${ }^{1}$, Yekun Zhang ${ }^{1}$, Hang Li ${ }^{\text {Corresp. } 1}$ \\ ${ }^{1}$ Key Laboratory of Soil Multi-Scale Interfacial Process, College of Resource and Environment, Southwest University, Chongqing, Chongqing, China \\ Corresponding Authors: Rui Tian, Hang Li \\ Email address: tr2016@swu.edu.cn, lihangswu@163.com
}

Background. The interactions between colloidal particles in the binary system or mixture colloids containing clay minerals and bacteria have important influences on formations and stabilities of soil aggregates, transportations of soil water, as well as biological activities of microorganisms. How the interfacial reaction of metal ions affects their interaction therefore becomes an important scientific issue.

Methods. Dynamic light scattering studies on the aggregation kinetics of mixture colloids containing kaolinite and Pseudomonas putida (P. putida) were conducted in this study.

Results. Aggregation could be observed between kaolinite and kaolinite, between kaolinite and P. putida when $P$. putida content was less than $33.3 \%$. Additionally, aggregation rates decreased with increasing $P$. putida content. The critical coagulation concentrations and activation energies indicated that there were strong specific ion effects on the aggregation of mixture colloids. Most importantly, the activation energy increased sharply with increasing $P$. putida content, which might result from the lower Hamaker constant of $P$. putida compared with that of kaolinite.

Contributions. (1) Strong specific ion effects on mixture colloids aggregation of kaolinite-Pseudomonas putida were observed; (2) the aggregation behavior of mixture colloidal system was determined by the average effects of mixture colloids, rather than the specific component in mixture systems. This finding provides an important methodological guide for further studies on the colloidal aggregation behavior of mixture systems with organic and inorganic materials. 
1 Aggregation kinetics of binary systems containing

2 kaolinite and Pseudomonas putida induced by

3 different 1:1 electrolytes: Specific ion effects

4

6 Zhaoxuan $\mathrm{Yu}^{1}$, Rui Tian ${ }^{1}$, Dian Liu, Yekun Zhang ${ }^{1}$, and Hang Li ${ }^{1}$

7

8 Key Laboratory of Soil Multi-Scale Interfacial Process, College of Resource and Environment,

9 Southwest University, Chongqing, Chongqing, China

11 Corresponding Author:

12 Rui Tian, Hang Li

13 No. 2 Tiansheng Road, Beibei District, Chongqing, China

14 Email address: R. Tian E-mail: tr2016@swu.edu.cn; H. Li: lihangswu@163.com 


\section{Abstract}

17 Background. The interactions between colloidal particles in the binary system or mixture colloids containing 18 clay minerals and bacteria have important influences on formations and stabilities of soil aggregates, 19 transportations of soil water, as well as biological activities of microorganisms. How the interfacial reaction of 20 metal ions affects their interaction therefore becomes an important scientific issue.

21 Methods. Dynamic light scattering studies on the aggregation kinetics of mixture colloids containing kaolinite and Pseudomonas putida (P. putida) were conducted in this study.

23 Results. Aggregation could be observed between kaolinite and kaolinite, between kaolinite and $P$. putida when 24 P. putida content was less than 33.3\%. Additionally, aggregation rates decreased with increasing P. putida content. The critical coagulation concentrations and activation energies indicated that there were strong specific ion effects on the aggregation of mixture colloids. Most importantly, the activation energy increased sharply with increasing $P$. putida content, which might result from the lower Hamaker constant of $P$. putida compared with that of kaolinite.

29 Contributions. (1) Strong specific ion effects on mixture colloids aggregation of kaolinite-Pseudomonas

30 putida were observed; (2) the aggregation behavior of mixture colloidal system was determined by the average

31 effects of mixture colloids, rather than the specific component in mixture systems. This finding provides an

32 important methodological guide for further studies on the colloidal aggregation behavior of mixture systems

33 with organic and inorganic materials. 
35

\section{Introduction}

Colloids in soil can be divided into inorganic colloids represented by minerals as well as organic colloids represented by microorganisms and organic macromolecules (Xiong 1983). The interactions between soil colloidal particles have important influences on the formation and stability of soil aggregates, the transportation of soil water, the biological activities of microorganisms, and so on (Borgnino 2013; Loosli et al. 2019). Dynamic light scattering (DLS) technique has been widely used in the study with respect to aggregation processes of soil mineral and organic colloids (Artemyeva et al. 2017; Derrendinger \& Sposito 2000; Nguyen et al. 2013; Nguyen et al. 2017; Tian et al. 2014; Yan et al. 2019; Zhu et al. 2017). Moreover, parameters with regarding to aggregation kinetics including the critical coagulation concentration (CCC) (Jia et al. 2013), aggregation activation energy (Tian et al. 2014), ionic polarization (Gao et al. 2014), and Hamaker constant (Luo et al. 2018) can be obtained by DLS measurement based on the determination of the hydrodynamic diameter of aggregates.

Recent studies have indicated that there is strong specific ion or Hofmeister effects on soil colloids aggregation. Based on the DLS study on the aggregation of montmorillonite colloids in the various alkali ion solutions, Tian et al. (2014)found that the $\mathrm{CCC}$ values for $\mathrm{Li}^{+}, \mathrm{Na}^{+}, \mathrm{K}^{+}, \mathrm{Rb}^{+}$ and $\mathrm{Cs}^{+}$(nitrate) were respectively 277.2, 132.8, 80.3, 31.7 and $27.2 \mathrm{mmol} / \mathrm{L}$. For the aggregation of humic acid and "mineral-humic acid" mixture colloids in the divalent ion solutions, similar ion specific effects were also observed (Gao et al. 2012; Gao et al. 2015). It has long been believed that the difference between ionic radius or ionic hydration radius should be the primary origin of specific ion effects (DeWalt-Kerian et al. 2017). However, compared with the difference from 
ionic electrostatic effects, the difference from ionic radius and ionic hydration radius is merely a quatratic term (Chen 2001), which cannot be used to interpret the above-mentioned up-to-10 times differences between $\mathrm{CCC}$ values. Recent study indicated that the ionic dispersion forces might be

an important reason for the specific ion effects (Bostrom et al. 2001), which could be important

especially when the Coulomb/electrostatic effect was weak (i.e., when the electrostatic field was

sufficiently shielded because of high electrolyte concentrations) (Parsons et al. 2011). However,

Tian et al. (2014) observed more pronounced specific ion effects at lower electrolyte concentrations, which obviously cannot be explained by the dispersion forces. A series of studies on the effects of ion interfacial reaction on colloidal particle aggregation have been carried out recently (Du et al. 2017; Gao et al. 2014; Hu et al. 2015; Li et al. 2015; Liu et al. 2014; Tian et al. 2015; Xu et al. 2015). They suggested that the strong non-classical polarization, which was caused by the strong electric field formed by the surface charge of clay minerals in water system, could give a rational explanation for specific ion effects on mineral colloids aggregation. These new studies indicated that this non-classical polarization effects could be as strong as the Coulomb force, and were up to $10^{4}$ times that of the classical polarization (Liu et al. 2014). Moreover, this non-classical polarization not only greatly enhanced the adsorption intensity of the ions onto the surface, but also deeply affected the interaction between the colloidal particles (Du et al. 2017). microorganisms is larger than the scale defined by colloidal particles, their colloidal properties are still obvious since the particle size of the microorganisms would be $1-1000 \mathrm{~nm}$ in at least one 
77 microorganisms as microbial colloids (Peng et al. 2018; Pisarcik et al. 2016). It's worth noting that

78 microbial colloids are neither mineral colloids nor soil humus colloids. Take the bacterial cell as

79 an example, a large number of functional groups and surface charges, existing on both interior and

80 exterior hydrophilic surfaces, have similarities with those generating from the general organic

81 macromolecules and even mineral colloidal surfaces; however, there is a lipid bilayer existing

82 between the interior and exterior surfaces of the bacterial cell membrane.

83 There have been plenty of studies on "mineral-bacteria" interactions so far (Diao et al. 2014;

84 Hong et al. 2014; Qu et al. 2018; Wu et al. 2012; Zhao et al. 2018; Zhao et al. 2012; Zhao et al.

85 2014), but the effects of ionic polarization, especially non-classical ionic polarization, on the

86 "mineral-bacteria" interaction are rarely reported. A large number of studies have shown that

87 addition of natural organic matter (NOM) leads to changes in the aggregation of inorganic colloids

88 (Ramirez et al. 2019; Sun et al. 2018). However, there is little study when it comes to

89 microorganisms. A large number of studies have shown that the addition of natural organic matter

90 leads to changes in the aggregation of inorganic colloids. It has been found that the adsorption of

91 bacteria onto the mineral surfaces is mainly affected by factors such as $\mathrm{pH}$, ionic strength (Wu et

92 al. 2012), clay type (Bellou et al. 2015), bacteria types and its cell surface properties (Poortinga et

93 al. 2002), bacteria growth cycle (Wu et al. 2014), bacteria/mineral mass ratio (Yee et al. 2000),

94 and so on (Tsagkari \& Sloan 2018). Many theories were used to explain these phenomena. The

95 attachment process of microorganisms onto minerals was described by the Langmuir isotherm

96 equation (Vasiliadou \& Chrysikopoulos 2011; Vasiliadou et al. 2011) and by the Freundlich

97 isotherm equation (Chrysikopoulos \& Syngouna 2012). Jiang et al. (2007) found that, compared 
with $\mathrm{Na}^{+}, \mathrm{Mg}^{2+}$ was more effective in promoting the adsorption of Pseudomonas putida (P. putida)

on minerals, indicating that electrostatic interaction plays an important role in the adsorption

process. Zhao et al. (2012) demonstrated that Derjaguin-Landau-Verwey-Overbeek (DLVO)

101 theory can be used to explain the adsorption of clay minerals (i.e., montmorillonite and kaolinite)

102 by Escherichia. coli and Streptococcus. suis under very low electrolyte conditions. The adsorption

103 of Bacillus subtilis on the phyllosilicates surface could be well explained by the extended DLVO

104 theory (Hong et al. 2014). Parikh and Chorover (2006) found that P-OFe covalent bonds can be

105 formed between the carboxyl or phosphate groups on the bacteria (P. putida) surface and the iron

106 atoms on the surface of hematite and goethite. Meanwhile, the adsorption thermodynamic

107 principles have been widely used in the study on "mineral-bacteria" interactions (Chen et al. 2003;

108 Chen \& Zhu 2005; Hendricks et al. 1979).

109 The above analysis indicates that it would be a valuable scientific question to clarify the role

110 of non-classical ionic polarization on the "mineral-bacteria" interaction. In this work, the

111 aggregation behaviors of "kaolinite- $P$. putida" induced by $\mathrm{LiNO}_{3}, \mathrm{KNO}_{3}$ and $\mathrm{CsNO}_{3}$ were

112 studied by D LS technique. Whether the aggregation of "mineral-bacteria" mixture colloids would

113 be affected by the specific ion effects was identified firstly, and then how the non-classical ionic

114 polarization effects affect the "mineral-bacteria" interaction was clarified.

$116 \quad$ Materials and Methods

117 Bacterial suspension

118 P. putida (Gram-negative), purchased from China Center for Type Culture Collection, was 
119 selected as the test strains. The strain P. putida was cultured at $30{ }^{\circ} \mathrm{C}$ on Luria-Bertani (LB) liquid

120 medium. The LB medium per liter $(\mathrm{pH}=7)$ contained $10 \mathrm{~g}$ Tryptone, $5 \mathrm{~g}$ Yeast and $10 \mathrm{~g} \mathrm{NaCl} . P$.

121 putida was stored in a glycerol tube $(30 \%)$ at $-80{ }^{\circ} \mathrm{C}$, and activated in $\mathrm{LB}$ medium at $30{ }^{\circ} \mathrm{C}$ and

$122120 \mathrm{r} / \mathrm{min}$ for $7 \mathrm{~h}$ until an optical density at $600 \mathrm{~nm}\left(\mathrm{OD}_{600}\right)$ of 0.5 was reached before the

123 experiment. $1 \mathrm{~mL}$ of the activation solution was incubated in $250 \mathrm{~mL} \mathrm{LB}$ medium at $30^{\circ} \mathrm{C}, 120$

$124 \mathrm{r} / \mathrm{min}$ for 14 hours when the cell reached the stationary phase $\left(\mathrm{OD}_{600}=2.0\right)$. Then the suspension

125 was freeze-dried (Dong, Zhou 2019). During the aggregation experiments, $10 \mathrm{mg}$ of freeze-dried

126 P. putida powders were identically dispersed in the $\mathrm{pH}=8.0$ sterile water (pre-adjusted by 10

$127 \mathrm{mmol} / \mathrm{L} \mathrm{NaOH}$ ). The bacterial suspension was ultrasonically dispersed with a KQ5200DE

128 ultrasonic disruptor at $40 \mathrm{kHz}$ for $15 \mathrm{~min}$. The hydrodynamic diameter of $P$. putida was measured

129 as $1600 \pm 100 \mathrm{~nm}$ through dynamic light measurement before adding electrolyte.

$130 \quad$ Kaolinite colloidal suspension

131 Kaolinite ( $>99.99 \%$ pure, purchased from Xuzhou, Jiangsu Province, China) was used in this

132 study. The cation exchange capacity (CEC) and specific surface area were determined to be 37.5

133 mequiv. $/ \mathrm{kg}$ and $57 \quad \mathrm{~m}^{2} / \mathrm{g}$, respectively. The kaolinite colloidal suspension

134 were prepared according to the following procedure (Xiong 1983). $50.0 \mathrm{~g}$ kaolinite particles and

$13510 \mathrm{~mL} 100 \mathrm{mmol} / \mathrm{L} \mathrm{KOH}$ solutions were successively added into a $500 \mathrm{~mL}$ beaker, and then diluted

136 with sterile water to $500 \mathrm{~mL}$. After $15 \mathrm{~min}$ of intensive sonication, the suspension was further

137 diluted to $5 \mathrm{~L}$ using sterile water. The kaolinite colloidal particles with the effective hydrodynamic

138 diameter of less than $300 \mathrm{~nm}$ were extracted and collected using the static sedimentation method.

139 The particle density was estimated to be $7.35 \mathrm{~g} / \mathrm{L}$ by the oven drying method. Then the prepared 
140 kaolinite colloidal suspensions were diluted 10 times and the $\mathrm{pH}$ value was measured to be around

141 8.0. The hydrodynamic diameter of kaolinite was measured to be $320 \pm 20 \mathrm{~nm}$.

\section{$142 \quad$ Experimental conditions}

143 The experiment was carried out at a temperature of $25^{\circ} \mathrm{C}$ and $\mathrm{pH}=8.0$, which can keep the

144 dispersion of both the bacteria and the kaolinite colloids as well as the cell integrity of the bacteria.

145 The selected electrolytes and their concentrations were $\mathrm{LiNO}_{3}(0-900 \mathrm{mmol} / \mathrm{L}), \mathrm{KNO}_{3}(0-700$

$146 \mathrm{mmol} / \mathrm{L})$, and $\mathrm{CsNO}_{3}(0-450 \mathrm{mmol} / \mathrm{L})$, respectively. The total mass concentration of the colloidal

147 suspension were $300 \mathrm{mg} / \mathrm{L}$, and the mass concentrations of the bacteria in the mixed suspension

148 were set as $0,10,20,50$, and $100 \mathrm{mg} / \mathrm{L}$, respectively, indicating the $P$. putida contents were $0 \%$,

$1493.33 \%, 6.67 \%, 16.67 \%$, and $33.33 \%$, respectively.

Dynamic light scattering measurement

151 A BI-200SM multi-angle laser light scattering instrument (Brookhaven Instruments Corporation,

152 New York, USA) with a BI-9000AT auto-correlator (Brookhaven Instruments Corporation) was

153 used for the measurement of the aggregates' particle size (e.g., effective hydrodynamic diameter)

154 which will change during the aggregation process. The power of the laser device equals $15 \mathrm{~mW}$

155 and the laser is vertically polarized with a wavelength of $532 \mathrm{~nm}$. Experimentally, ultrapure water,

156 kaolinite suspensions, bacteria suspensions and the electrolyte $\left(\mathrm{LiNO}_{3}, \mathrm{KNO}_{3}\right.$ or $\left.\mathrm{CsNO}_{3}\right)$ solutions

157 of different concentrations were mixed in the scattering bottle with the total volume of $10 \mathrm{~mL}$. The

158 procedure of aggregation kinetic measurement was that: each colloidal (including single and mixed

159 colloids) suspension was prepared with a 2 min sonication before adding the electrolyte. After

160 adding electrolyte, the information about particles size and the size distribution was recorded 
161 automatically by DLS measurement. The duration for each experiment was $30 \mathrm{~min}$. We chose 30

162 min to be the duration time, because after 30 min aggregation the light scattering intensity

163 gradually became unstable.

164 CCC, the minimum electrolyte concentration required for the Diffusion-Limited colloid

165 aggregation (DLCA) regime (Lin et al. 1989), is an important parameter for characterizing the

166 colloidal aggregation. The CCC value directly reflects the difference in the colloidal stability.

167 Based on the research of Jia et al. (2013), the aggregation kinetics of colloidal particles in

168 electrolyte solutions can be described by the total average aggregation (TAA) rate, which was

169 expressed as,

$$
\Re\left(f_{0}\right)=\frac{1}{t_{0}} \int_{0}^{t_{0}} \frac{D(t)-D_{0}}{t} d t
$$

171 where $\underset{v^{T}}{\sim}\left(f_{0}\right)(\mathrm{nm} / \mathrm{min})$ is the TAA rate from $t=0$ to a given time $t=t_{0}\left(t_{0}>0\right)$ which is committed

172 to a time limit of the aggregation process; $f_{0}(\mathrm{mmol} / \mathrm{L})$ is the electrolyte concentration; $D_{0}$ and $D_{(\mathrm{t})}$

$173(\mathrm{~nm})$ are respectively the effective hydrodynamic diameters of aggregates at the beginning and at

174 time $t_{0}$.

175 Tian et al. (2014) showed that the activation energy $(\Delta E)$ and the TAA rate $\left.\tilde{v_{v}^{T}}\left(f_{0}\right)\right)$ are

176 correlated with the expressions as

$$
\Delta E=-R T \ln \frac{\vartheta / \rho\left(f_{0}\right)}{\vartheta / P(C C C)}
$$

178 where, $R(\mathrm{~J} / \mathrm{mol} \mathrm{K})$ is the gas constant and $T(\mathrm{~K})$ is the absolute temperature.

179 Zeta potential measurements

180 The zeta potentials of "bacteria-kaolinite" mixture colloids as functions of the bacteria 
181 contents were measured in DI water using ZETA Plus (Brookhaven Instruments Corporation, New

182 York, USA) at $25^{\circ} \mathrm{C}, \mathrm{pH} 8.0$. The samples for the zeta potential measurements were prepared in a

183 similar manner as those for the aggregation experiments. The total mass concentration of the

184 sample suspension was $300 \mathrm{mg} / \mathrm{L}$, and the mass concentrations of the bacteria in the mixed

185 suspension were set as $0,10,20,50$, and $100 \mathrm{mg} / \mathrm{L}$, respectively. Triplicate measurements were

186 performed with ten runs per measurement.

187 Results and discussion

$188 \quad$ Kinetics of "kaolinite-P. putida" aggregation

189 The curves describing the hydrodynamic diameter growth of "kaolinite- $P$. putida" mixture

190 colloids in $\mathrm{LiNO}_{3}$ solutions were shown in Fig. 1. It could be seen that, the hydrodynamic

191 diameters of "kaolinite- $P$. putida" aggregates increased with increasing $\mathrm{LiNO}_{3}$ concentrations. For

192 example, the hydrodynamic diameters of mixture colloids containing $100 \%$ kaolinite and $0 \% P$.

193 putida indicated that aggregation occurred between kaolinite and kaolinite. However, the

194 hydrodynamic diameter of the mixture colloids containing $0 \%$ kaolinite and $100 \%$ P. putida

195 suggested that aggregation would not occur between $P$. putida and $P$. putida. The mixture colloids

196 aggregated when the proportion of $P$. putida was $0 \%, 3.33 \%, 6.67 \%, 16.67 \%$ and $33.33 \%$, but the

197 aggregation rate decreased with increasing proportion of $P$. putida.

198 The curves describing the hydrodynamic diameters of different "kaolinite-P. putida" mixture

199 as a function of time in $\mathrm{KNO}_{3}$ solutions were shown in Fig. 2. Similar to the aggregation in $\mathrm{LiNO}_{3}$

200 solutions, the hydrodynamic diameters of "kaolinite- $P$. putida" mixture colloidal aggregates

201 increased with increasing $\mathrm{KNO}_{3}$ concentrations. Additionally, the aggregation can occur between 
202 kaolinite and kaolinite but not occur between $P$. putida and $P$. putida. The mixture colloids

203 aggregated when the proportion of $P$. putida was $0 \%, 3.33 \%, 6.67 \%, 16.67 \%$ and $33.33 \%$, but the

204 aggregation rate decreased with increasing proportion of $P$. putida.

205 The curves describing the hydrodynamic diameter of different proportions of "kaolinite- $P$.

206 putida" as a function of time in $\mathrm{CsNO}_{3}$ were shown Fig. 3. Similar to colloidal aggregation in

$207 \mathrm{LiNO}_{3}$ and $\mathrm{KNO}_{3}$ solutions, the hydrodynamic diameters of "kaolinite- $P$. putida " mixture

208 colloidal aggregates increased with the increase of $\mathrm{CsNO}_{3}$ concentration. The aggregation can

209 occur between kaolinite and kaolinite but not between P. putida and P. putida.

$210 \quad$ TAA rates and CCC

211 Using the experimental data given in Figs. 1, 2, and 3, the TAA rates ${ }_{v^{T}}^{\sim}\left(f_{0}\right)$ of mixture colloids

212 aggregation in various alkali ion solutions were calculated according to Eq. (1), and their

213 relationships with the alkali ion concentration $f_{0}$ were given in Table 1 . The more detailed results

214 of the TAA rates changing with electrolyte concentrations has been given in the Supporting

215 Information (Figs. S1 S5).

216 As can be seen, the CCC of the mixture colloids under different bacterial contents can be

217 obtained (Table 1). For any given electrolyte solutions, the CCC for the mixture colloids increased

218 as the bacterial content increased. For the aggregation of the mixture colloids with the same

219 bacterial contents, the CCC increased in the order of $\mathrm{Li}^{+}>\mathrm{K}^{+}>\mathrm{Cs}^{+}$, exhibiting strong specific ion

220 effects. For example, when the $P$. putida content was $3.33 \%$, the CCCs for the aggregation of

221 mixture colloids were 18.7,16.7 and $9.4 \mathrm{mmol} / \mathrm{L}$ for $\mathrm{Li}^{+}, \mathrm{K}^{+}$and $\mathrm{Cs}^{+}$, respectively. Moreover, the

222 specific ion effects reflected by the CCC values increased as the bacterial content increased. 
When the electrolyte concentrations were less than the CCC, the aggregation belongs to the

224 RLCA regime, and the repulsive potential energy between the colloidal particles was higher than

225 the attractive potential energy. Table 1 showed the fitting equation for aggregation of "kaolinite-

226 P. putida" mixture colloids in different electrolyte solutions when the electrolyte concentrations

227 were less than the CCC. As can be seen, given the same electrolyte conditions, the TAA rates

228 of "kaolinite- $P$. putida" mixture colloids decreased as the bacterial content increased. On the other

229 hand, for the same bacterial contents, the TAA rates for the aggregation of "kaolinite-P. putida"

230 mixture colloids increased in the order of $\mathrm{Li}^{+}<\mathrm{K}^{+}<\mathrm{Cs}^{+}$, exhibiting strong specific ion effects.

231 For example, when the $P$. putida content was $3.33 \%$, the TAA rates for the aggregation of mixture

232 colloids were $1.21,48.2$, and $80.5 \mathrm{~nm} / \mathrm{min}$ for $\mathrm{Li}^{+}, \mathrm{K}^{+}$and $\mathrm{Cs}^{+}$, respectively.

233 Interaction between $\boldsymbol{P}$. putida and kaolinite and the aggregation mechanism

234 The activation energies for the aggregation of the mixture colloids in the various alkali ion

235 solutions were calculated using Eq. 2 and plotted in Fig. 4. At any given same electrolyte

236 concentrations below CCC, the activation energies for the mixture colloids with different bacteria

237 contents were significantly different. In addition, the activation energies increased as the bacterial

238 content increased. At any given different alkali ion concentrations below CCC, the activation

239 energies for the mixture colloids with the same bacteria contents increased in the order of $\mathrm{Li}^{+}>\mathrm{K}^{+}$

$240>\mathrm{Cs}^{+}$. For example, when the $P$. putida content was $3.33 \%$ and the electrolyte concentration was

$2415 \mathrm{mmol} / \mathrm{L}$, the activation energy for the aggregation of mixture colloids were respectively $1.5 R T$,

$2421.4 R T$, and $0.7 R T$ for $\mathrm{Li}^{+}, \mathrm{K}^{+}$and $\mathrm{Cs}^{+}$.

243 The activation energies for the mixture colloids aggregation might come from the electrostatic 
244 repulsion potential as well as the molecular attraction potential between P. putida and kaolinite.

245 The electrostatic repulsion potential could be characterized by the zeta potential, we therefore

246 determined the zeta potential of "kaolinite- $P$. putida " mixture colloids, and the results were shown

247 in Fig. 5. The Zeta potential of kaolinite at $\mathrm{pH}=8$ is $-44 \mathrm{mv}$, which is not far from the $-46 \mathrm{mv}$

248 reported by Feng et al. (2020) at $\mathrm{pH}=7$. However, The Zeta potential of $P$. putida at $\mathrm{pH}=8$ is -37

$249 \mathrm{mv}$, which is far from the $-8.5 \mathrm{mv}$ reported by Shamim and Rehman (2014) at $\mathrm{pH}=6.2 \mathrm{We}$ suspect

250 that it may be related to $\mathrm{pH}$ and the pretreatment process of bacteria.

251 From Fig. 5, we could see that the electrostatic repulsion potential between $P$. putida and

252 kaolinite decreased as the $P$. putida content increased. In other words, the activation energy for the

253 mixture collolds aggregation should decrease as the $P$. putida content increased. According to the

254 experimental results shown in Fig. 4, the activation energy for the mixture collolds aggregation,

255 however, increased with the increase of $P$. putida content. Therefore, the only possible explanation

256 for the decrease in the aggregation rate with increasing bacterial content could be that the Hamaker

257 constant of the bacteria is significantly lower than that of kaolinite. In this case, the apparent

258 Hamaker constant of the mixture colloids decreased with increasing P. putida content, which

259 would further lead to the decrease of molecular attractive energies between colloidal particles.

260 However, apart from the aggregation between $P$. putida and kaolinite in the mixture colloids,

261 another two kinds of aggregation process might occur: the aggregation between $P$. putida and $P$.

262 putida as well as the aggregation between kaolinite and kaolinite. We can speculate that there may

263 be three CCCs in the mixture colloids: the CCC of kaolinite-kaolinite aggregation, the CCC of P.

264 putida-P. putida aggregation and the CCC of kaolinite-P. putida aggregation. According to the 
265 experimental results, we can find that, the CCC for pure kaolinite colloids (kaolinite-kaolinite 266 aggregation) is the lowest; the CCC for $100 \%$ P. putida colloids (P. putida-P. putida aggregation)

267 is infinite. Therefore, we can speculate that the CCC for kaolinite-P. putida aggregation would be

268 in the middle. Therefore, based on this analysis, at a given $P$. putida content and a given cation

269 type, the variation of TAA rates changing with electrolyte concentrations for the mixture colloids

270 should exhibit the characteristics as shown in Fig. 6. The first straight line at low electrolyte

271 concentrations should represent the aggregation between kaolinite and kaolinite because the

272 activation energy for the aggregation between kaolinite and kaolinite is the lowest. The activation

273 energy for the mixture colloids greatly reduced when the electrolyte concentration gradually

274 increased higher than the first turning point. The aggregation of $P$. putida and kaolinite became

275 important. Therefore, the second straight line contained the rapid aggregation of kaolinite and

276 kaolinite and the slow aggregation of $P$. putida and kaolinite. The third straight line represented

277 the rapid aggregation of $P$. putida and kaolinite. Therefore, the electrolyte concentration

278 corresponding to the second inflection point is the CCC of P. putida and kaolinite.

279 However, in the mixture colloids, just one CCC exhibited for given mixture suspension, and

280 for different mixture suspensions, the CCC increased as the content of $P$. putida increased (Table

281 1). Table 1 showed that, (1) the two turning points shown in Fig. 6 could not be observed from the

282 experimental results of the $P$. putida and kaolinite mixture colloids. (2) The CCC of kaolinite and

283 kaolinite was not observed from the mixture colloids. (3) There is only one CCC obtained for the

284 mixture colloids. Based on this, we can deduce that the aggregation behavior of the mixture

285 colloids is determined by the average effect produced by the various colloidal particles instead of 
separate colloidal particle. The experimental results shown in Table 1 could be satisfactorily

287 explained by this inference. Each mixture colloidal system can only have one specific CCC, which

288 is determined by each physical parameter (such as determination of the average value of potential,

289 electric field strength, charge density, Hamaker constant, etc.) since each physical parameter of

290 the mixed system (such as potential, electric field strength, charge density, Hamaker constant, etc.)

291 has only one average value. What's more, the average Hamaker constant of the mixture colloids

292 decreased with the increase of $P$. putida content due to higher Hamker constant of kaolinite and

293 lower Hamaker constant of P. putida. Eventually, the CCC of mixed system increases with the

294 increase of $P$. putida content.

295 Finally, we would like to give a speculated explanation for the experimental results for the

296 kinetics of pure $P$. putida aggregation. We know that, $P$. putida is a gram-negative, rod-shaped

297 bacteria, and with the polar flagellum or with positively and negatively charges at cell surfaces; in

298 addition, generally, bacteria prefer to survive in cell groups but not a single cell. Therefore, $P$.

299 putida could aggregate easily. In our experiments, however, (1) the net charge of $P$. putida was

300 negative, therefore, the electrostatic fore between two cells must be repulsive as the distance

301 between two cells was relative long; (2) the adopted cations in this study were monovalent cation,

302 those cations just could produce relative weak screening effect on the electric field around cell,

303 thus a long range electrostatic repulsive force between two cells could present; (3) the density of

304 cell particles in this experiments was very low, thus the long range electrostatic repulsive force

305 dominate cells interaction. Those three points might be the reasons for explaining the kinetics of

306 pure P. putida aggregation. Therefore, on the contrary, if the cell density was very high, and if 
307 divalent cation presented in the suspension, the cell aggregation might be possibly observed.

$308 \quad$ Conclusions

309 In this paper, the aggregation behaviors of $P$. putida, kaolinite and "kaolinite- $P$. putida"

310 mixed colloids in various concentrations of $\mathrm{LiNO}_{3}, \mathrm{KNO}_{3}$ and $\mathrm{CsNO}_{3}$ electrolytes were studied.

311 Aggregation occured between kaolinite and kaolinite whereas no aggregation occured between $P$.

312 putida and $P$. putida in the presence of any kind of the three electrolytes. Additionally, the

313 aggregation occured in the mixture colloids when the bacterial content in the mixed system was

314 less than 50\%. TAA rate decreased with the increasing bacterial content. Additionally, specific ion

315 effects affected the aggregation of "P. putida -kaolinite" mixture colloids. The TAA rates for the

316 aggregation of the mixture colloids with same bacteria content increased in the order of $\mathrm{Cs}^{+}>\mathrm{K}^{+}$

$317>\mathrm{Li}^{+}$. For example, when the $P$. putida content equaled $3.33 \%$, the TAA rates for the aggregation

318 of the mixture colloids were $1.21,48.2,80.5 \mathrm{~nm} / \mathrm{min}$ for $\mathrm{Li}^{+}, \mathrm{K}^{+}$and $\mathrm{Cs}^{+}$respectively. The CCC

319 for the aggregation of the mixture colloids with the same bacteria content increased in the order of

$320 \mathrm{Li}^{+}>\mathrm{K}^{+}>\mathrm{Cs}^{+}$. The CCC for the aggregation of the mixture colloids were 18.7, 16.7 and 9.4

$321 \mathrm{mmol} / \mathrm{L}$ for $\mathrm{Li}^{+}, \mathrm{K}^{+}$and $\mathrm{Cs}^{+}$, respectively, when the $P$. putida content equaled $3.33 \%$. The

322 aggregation activation energy for the aggregation of the mixture colloids with the same bacteria

323 contents increased in the order $\mathrm{Li}^{+}>\mathrm{K}^{+}>\mathrm{Cs}^{+}$. The increasing of aggregation activation energy

324 and decreasing of TAA rates for the aggregation of the mixture colloids as $P$. putida content

325 increased might come from the lower Hamaker constant of $P$. putida than that of kaolinite, which

326 further gave rise to lower molecular gravitational potential between colloidal particles. What's

327 more, the aggregation behavior of a mixed colloidal system was determined by the average effect 
328 of electrostatic potential and molecular gravitational potential produced by the various colloidal

329 particles instead of separate colloidal particle. This finding provides an important methodological

330 guide for studying the aggregation behavior of "bacterial-clay" mixed systems.

\section{$331 \quad$ Acknowledgements}

This work was supported by the National Natural Science Foundation of China (grant numbers

41501241 and 41530855); Fundamental Research Funds for the Central Colleges (grant number

334

335

336

337

338

339

340

341

342

343

344

345

346

347

348

349

350

351

352

353

354

355

356

357

358

359
XDJK2019B037).

\section{References}

Artemyeva Z, Zigova A, Kirillova N, Stastny M, Holubik O, and Podrazsky V. 2017. Evaluation of aggregate stability of Haplic Stagnosols using dynamic light scattering, phase analysis light scattering and color coordinates. Archives of Agronomy and Soil Science 63:1838-1851. 10.1080/03650340.2017.1311012

Bellou MI, Syngouna VI, Tselepi MA, Kokldnos PA, Paparrodopoulos SC, Vantarakis A, and Chrysikopoulos CV. 2015. Interaction of human adenoviruses and coliphages with kaolinite and bentonite. Science Of the Total Environment 517:86-95. 10.1016/j.scitotenv.2015.02.036

Borgnino L. 2013. Experimental determination of the colloidal stability of Fe(III)-montmorillonite: Effects of organic matter, ionic strength and pH conditions. Colloids And Surfaces A-Physicochemical And Engineering Aspects 423:178-187. 10.1016/j.colsurfa.2013.01.065

Bostrom M, Williams DRM, and Ninham BW. 2001. Specific ion effects: Why DLVO theory fails for biology and colloid systems. Physical Review Letters 87:4. 10.1103/PhysRevLett.87.168103

Chen G, Rockhold M, and Strevett KA. 2003. Equilibrium and kinetic adsorption of bacteria on alluvial sand and surface thermodynamic interpretation. Research In Microbiology 154:175-181. 10.1016/s09232508(03)00033-0

Chen G, and Zhu HL. 2005. Bacterial adhesion to silica sand as related to Gibbs energy variations. Colloids And Surfaces B-Biointerfaces 44:41-48. 10.1016/j.colsurfb.2005.05.012

Chen Z. 2001. Colloid and interface chemistry. Beijing China: Higher Education Press.

Chrysikopoulos CV, and Syngouna VI. 2012. Attachment of bacteriophages MS2 and Phi X174 onto kaolinite and montmorillonite: Extended-DLVO interactions. Colloids And Surfaces B-Biointerfaces 92:7483. 10.1016/j.colsurfb.2011.11.028

Derrendinger L, and Sposito G. 2000. Flocculation kinetics and cluster morphology in illite $/ \mathrm{NaCl}$ suspensions. Journal Of Colloid And Interface Science 222:1-11. 10.1006/jcis.1999.6606

DeWalt-Kerian EL, Kim S, Azam MS, Zeng HB, Liu QX, and Gibbs JM. 2017. pH-Dependent inversion 
of Hofmeister trends in the water structure of the electrical double layer. Journal Of Physical Chemistry Letters 8:2855-2861. 10.1021/acs.jpclett.7b01005

362

363

Diao MX, Taran E, Mahler S, and Nguyen AV. 2014. A concise review of nanoscopic aspects of bioleaching bacteria-mineral interactions. Advances In Colloid And Interface Science 212:45-63. 10.1016/j.cis.2014.08.005

365

366

367

Du W, Li R, Liu X, Tian R, Ding W, and Li H. 2017. Estimating Hofmeister energy in ion-clay mineral interactions from the Gouy-Chapman theory. Applied Clay Science 146:122-130. 10.1016/j.clay.2017.05.039

Feng W, Li Y, Lin Z, Luo Y, Wang S, and Qiu R. 2020. The influence on biosorption potentials of

369

370

371

372

373

374

375

376

377

378

379

380

381

382

383

384

385

386

387

388

389

390

391

392

393

394

395

396

397

398

399

400 metal-resistant bacteria Enterobacter sp. EG16 and Bacillus subtilis DBM by typical red soil minerals. Journal Of Soils And Sediments. 10.1007/s11368-020-02650-y

Gao X, Li H, Lin H, and Tian R. 2012. Aggregation of humic colloidal particles as affeccted by electrolyte and pH (in chinese) . Acta Pedologica Sinica. 49:698-707. 10.11766/trxb201104180139

Gao XD, Li H, Tian R, Liu XM, and Zhu HL. 2014. Quantitative characterization of specific ion effects using an effective charge number based on the Gouy-Chapman model. Acta Physico-Chimica Sinica. 30:2272-2282. 10.3866/pku.whxb201410231

Gao XD, Yang G, Tian R, Ding WQ, Hu FN, Liu XM, and Li H. 2015. Formation of sandwich structure through ion adsorption at the mineral and humic interfaces: A combined experimental computational study. Journal Of Molecular Structure 1093:96-100. 10.1016/j.molstruc.2015.03.060

Hendricks DW, Post FJ, and Khairnar DR. 1979. Adsorption of bacteria on soils: Experiments, thermodynamic rationale, and application. Water Air And Soil Pollution 12:219-232. 10.1007/bf01047124

Hong ZN, Zhao G, Chen WL, Rong XM, Cai P, Dai K, and Huang QY. 2014. Effects of solution chemistry on bacterial adhesion with Phyllosilicates and goethite explained by the extended DLVO theory. Geomicrobiology Journal 31:419-430. 10.1080/01490451.2013.824523

Hu FN, Li H, Liu XM, Li S, Ding WQ, Xu CY, Li Y, and Zhu LH. 2015. Quantitative characterization of non-classic polarization of cations on clay aggregate stability. Plos One 10:19. 10.1371/journal.pone.0122460

Jia M, Li H, Zhu H, Tian R, and Gao X. 2013. An approach for the critical coagulation concentration estimation of polydisperse colloidal suspensions of soil and humus. Journal Of Soils And Sediments 13:325335. 10.1007/s11368-012-0608-8

Jiang D, Huang Q, Cai P, Rong X, and Chen W. 2007. Adsorption of Pseudomonas putida on clay minerals and iron oxide. Colloids And Surfaces B-Biointerfaces 54:217-221. 10.1016/j.colsurfb.2006.10.030

Li S, Li H, Hu FN, Huang XR, Xie DT, and Ni JP. 2015. Effects of strong ionic polarization in the soil electric field on soil particle transport during rainfall. European Journal Of Soil Science 66:921-929. 10.1111/ejss. 12273

Lin MY, Lindsay HM, Weitz DA, Ball RC, Klein R, and Meakin P. 1989. Universality in colloid aggregation. Nature 339:360-362. 10.1038/339360a0

Liu X, Hang L, Rui L, Xie D, Ni J, and Wu L. 2014. Strong non-classical induction forces in ion-surface interactions: General origin of Hofmeister effects. Scientific Reports 4:5047. 10.1038/srep05047

Loosli F, Yi ZB, Wang JJ, and Baalousha M. 2019. Improved extraction efficiency of natural nanomaterials in soils to facilitate their characterization using a multimethod approach. Science Of the Total 
401

402

403

404

405

406

407

408

409

410

411

412

413

414

415

416

417

418

419

420

421

422

423

424

425

426

427

428

429

430

431

432

433

434

435

436

437

438

439

440

441

Environment 677:34-46. 10.1016/j.scitotenv.2019.04.301

Luo Y, Gao X, Tian R, and Li H. 2018. Approach to estimation of hamaker constant as taking Hofmeister effects into account. Journal Of Physical Chemistry C 122:9432-9440. 10.1021/acs.jpcc.7b12830

Nguyen MN, Dultz S, Tran TTT, and Bui ATK. 2013. Effect of anions on dispersion of a kaolinitic soil clay: A combined study of dynamic light scattering and test tube experiments. Geoderma 209:209-213. 10.1016/j.geoderma.2013.06.024

Nguyen MN, Picardal F, Dultz S, Nguyen-Thanh L, Dam TTN, and Nguyen KM. 2017. Effect of silicic acid on the aggregation properties of goethite. European Journal Of Soil Science 68:650-657. 10.1111/ejss. 12453

Parikh SJ, and Chorover J. 2006. ATR-FTIR spectroscopy reveals bond formation during bacterial adhesion to iron oxide. Langmuir 22:8492-8500. 10.1021/la061359p

Parsons DF, Bostrom M, Lo Nostro P, and Ninham BW. 2011. Hofmeister effects: interplay of hydration, nonelectrostatic potentials, and ion size. Physical Chemistry Chemical Physics 13:12352-12367. $10.1039 / \mathrm{c} 1 \mathrm{cp} 20538 \mathrm{~b}$

Peng KM, Liu WQ, Xiong YJ, Lu LJ, Liu J, and Huang XF. 2018. Emulsion microstructural evolution with the action of environmentally friendly demulsifying bacteria. Colloids And Surfaces A-Physicochemical And Engineering Aspects 553:528-538. 10.1016/j.colsurfa.2018.06.005

Pisarcik M, Pupak M, Devinsky F, Almasy L, Tian Q, and Bukovsky M. 2016. Urea-based gemini surfactants: Synthesis, aggregation behaviour and biological activity. Colloids And Surfaces APhysicochemical And Engineering Aspects 497:385-396. 10.1016/j.colsurfa.2016.03.028

Poortinga AT, Bos R, Norde W, and Busscher HJ. 2002. Electric double layer interactions in bacterial adhesion to surfaces. Surface Science Reports 47:3-32. 10.1016/s0167-5729(02)00032-8

Qu C, Du H, Ma M, Chen W, Cai P, and Huang Q. 2018. Pb sorption on montmorillonite-bacteria composites: A combination study by XAFS, ITC and SCM. Chemosphere 200:427-436. 10.1016/j.chemosphere.2018.02.136

Ramirez L, Gentile SR, Zimmermann S, and Stoll S. 2019. Behavior of $\mathrm{TiO}_{2}$ and $\mathrm{CeO}_{2}$ nanoparticles and polystyrene nanoplastics in bottled mineral, drinking and lake Geneva waters. Impact of water hardness and natural organic matter on nanoparticle surface properties and aggregation. Water 11:14. 10.3390/w11040721

Shamim S, and Rehman A. 2014. Physicochemical surface properties of Cupriavidus metallidurans CH34 and Pseudomonas putida mt2 under cadmium stress. Journal Of Basic Microbiology 54:306-314. 10.1002/jobm.201200434

Sumner ME. 2000. Handbook of soil science. Boca Raton, USA: CRC Press.

Sun C, Zhang N, Li F, Ke G, Song L, Liu X, and Liang S. 2018. Quantitative analysis of membrane fouling mechanisms involved in microfiltration of humic acid-protein mixtures at different solution conditions. Water 10. 10.3390/w10101306

Tian R, Yang G, Li H, Gao XD, Liu XM, Zhu HL, and Tang Y. 2014. Activation energies of colloidal particle aggregation: towards a quantitative characterization of specific ion effects. Physical Chemistry Chemical Physics 16:8828-8836. 10.1039/c3cp54813a

Tian R, Yang G, Liu XM, Huang CZ, Gao XD, and Li H. 2015. Observation of the unusual aggregation 
442

443

444

445

446

447

448

449

450

451

452

453

454

455

456

457

458

459

460

461

462

463

464

465

466

467

468

469

470

471

472

473

474

475

476

477

478

kinetics of colloidal minerals in acidic solutions. Journal Of Chemical Sciences 127:1083-1089. 10.1007/s12039-015-0872-4

Tsagkari E, and Sloan WT. 2018. The role of the motility of methylobacterium in bacterial interactions in drinking water. Water 10. 10.3390/w10101386

Vasiliadou IA, and Chrysikopoulos CV. 2011. Cotransport of Pseudomonas putida and kaolinite particles through water-saturated columns packed with glass beads. Water Resources Research 47:14. 10.1029/2010wr009560

Vasiliadou IA, Papoulis D, Chrysikopoulos CV, Panagiotaras D, Karakosta E, Fardis M, and Papavassiliou G. 2011. Attachment of Pseudomonas putida onto differently structured kaolinite minerals: A combined ATR-FTIR and H-1 NMR study. Colloids And Surfaces B-Biointerfaces 84:354-359. 10.1016/j.colsurfb.2011.01.026

Wu H, Chen W, Rong X, Cai P, Dai K, and Huang Q. 2014. Adhesion of Pseudomonas putida onto kaolinite at different growth phases. Chemical Geology 390:1-8. 10.1016/j.chemgeo.2014.10.008

Wu HY, Jiang DH, Cai P, Rong XM, Dai K, Liang W, and Huang QY. 2012. Adsorption of Pseudomonas putida on soil particle size fractions: effects of solution chemistry and organic matter. Journal Of Soils And Sediments 12:143-149. 10.1007/s11368-011-0441-5

Xiong Y. 1983. Soil colloid. Beijing China: Science Press.

Xu CY, Li H, Hu FN, Li S, Liu XM, and Li Y. 2015. Non-classical polarization of cations increases the stability of clay aggregates: specific ion effects on the stability of aggregates. European Journal Of Soil Science 66:615-623. 10.1111/ejss.12252

Yan CR, Cheng T, and Shang JY. 2019. Effect of bovine serum albumin on stability and transport of kaolinite colloid. Water Research 155:204-213. 10.1016/j.watres.2019.02.022

Yee N, Fein JB, and Daughney CJ. 2000. Experimental study of the pH, ionic strength, and reversibility behavior of bacteria-mineral adsorption. Geochimica et Cosmochimica Acta 64:609-617. 10.1016/s00167037(99)00342-7

Zhao G, Li E, Li J, Xu M, Huang Q, and Rong X. 2018. Effects of interfaces of goethite and humic acidgoethite complex on microbial degradation of methyl parathion. Frontiers In Microbiology 9:1-8. 10.3389/fmicb.2018.01748

Zhao WQ, Liu X, Huang QY, Walker SL, and Cai P. 2012. Interactions of pathogens Escherichia coli and Streptococcus suis with clay minerals. Applied Clay Science 69:37-42. 10.1016/j.clay.2012.07.003

Zhao WQ, Walker SL, Huang QY, and Cai P. 2014. Adhesion of bacterial pathogens to soil colloidal particles: Influences of cell type, natural organic matter, and solution chemistry. Water Research 53:35-46. 10.1016/j.watres.2014.01.009

Zhu L, Tian R, Liu X, Xiong H, and Li H. 2017. A general theory for describing coagulation kinetics of variably charged nanoparticles. Colloids And Surfaces A-Physicochemical And Engineering Aspects 527:158-163. 10.1016/j.colsurfa.2017.04.021

Peer] Phy. Chem. reviewing PDF | (PCHEM-2020:03:47222:1:2:NEW 4 Aug 2020) 


\section{Figure 1}

Figure. 1 Growth of hydrodynamic diameters of "kaolinite-P. putida" aggregates in $\mathrm{LiNO}_{3}$ solutions 

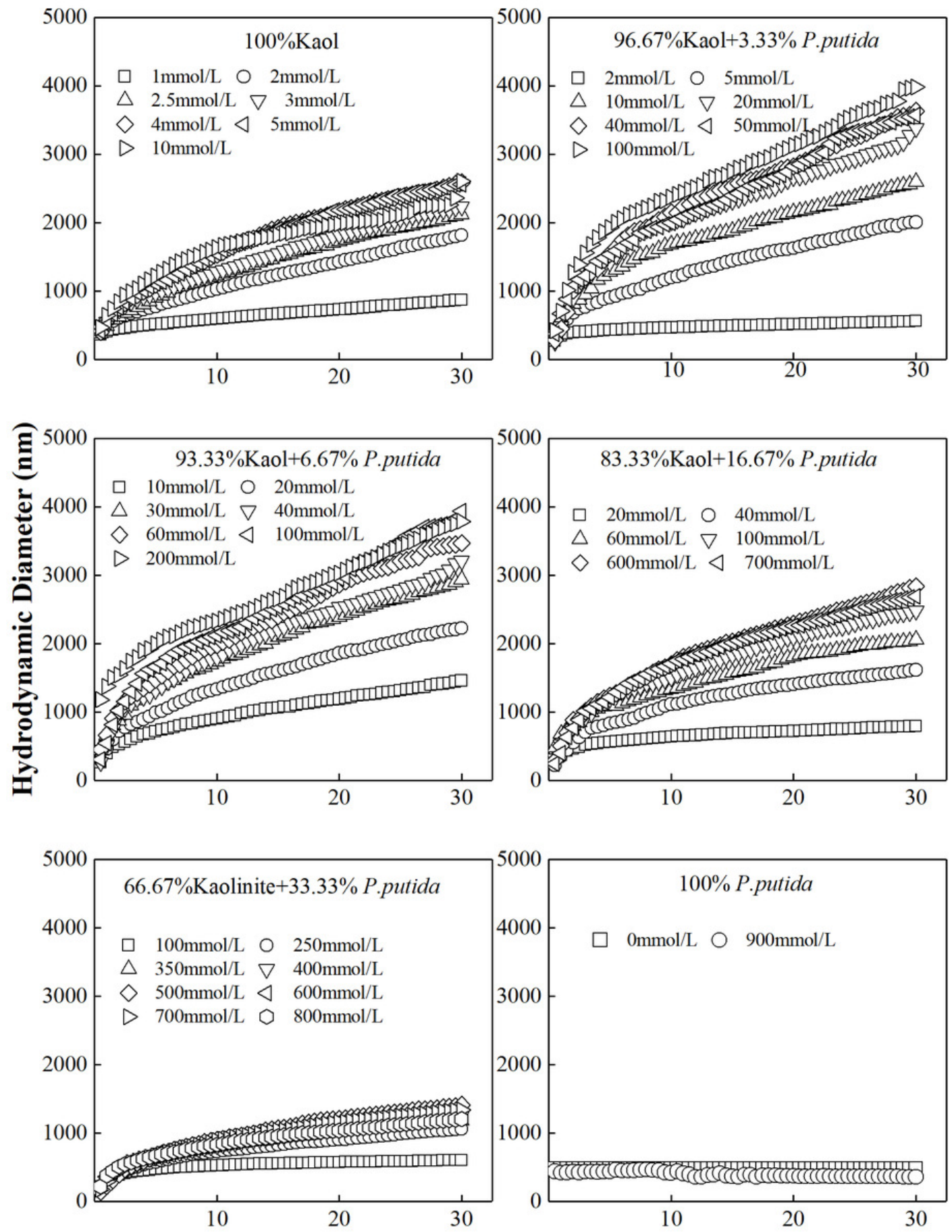

Time (min) 


\section{Figure 2}

Figure. 2 Growth of hydrodynamic diameters of "kaolinite-P. putida" aggregates in $\mathrm{KNO}_{3}$ solutions 

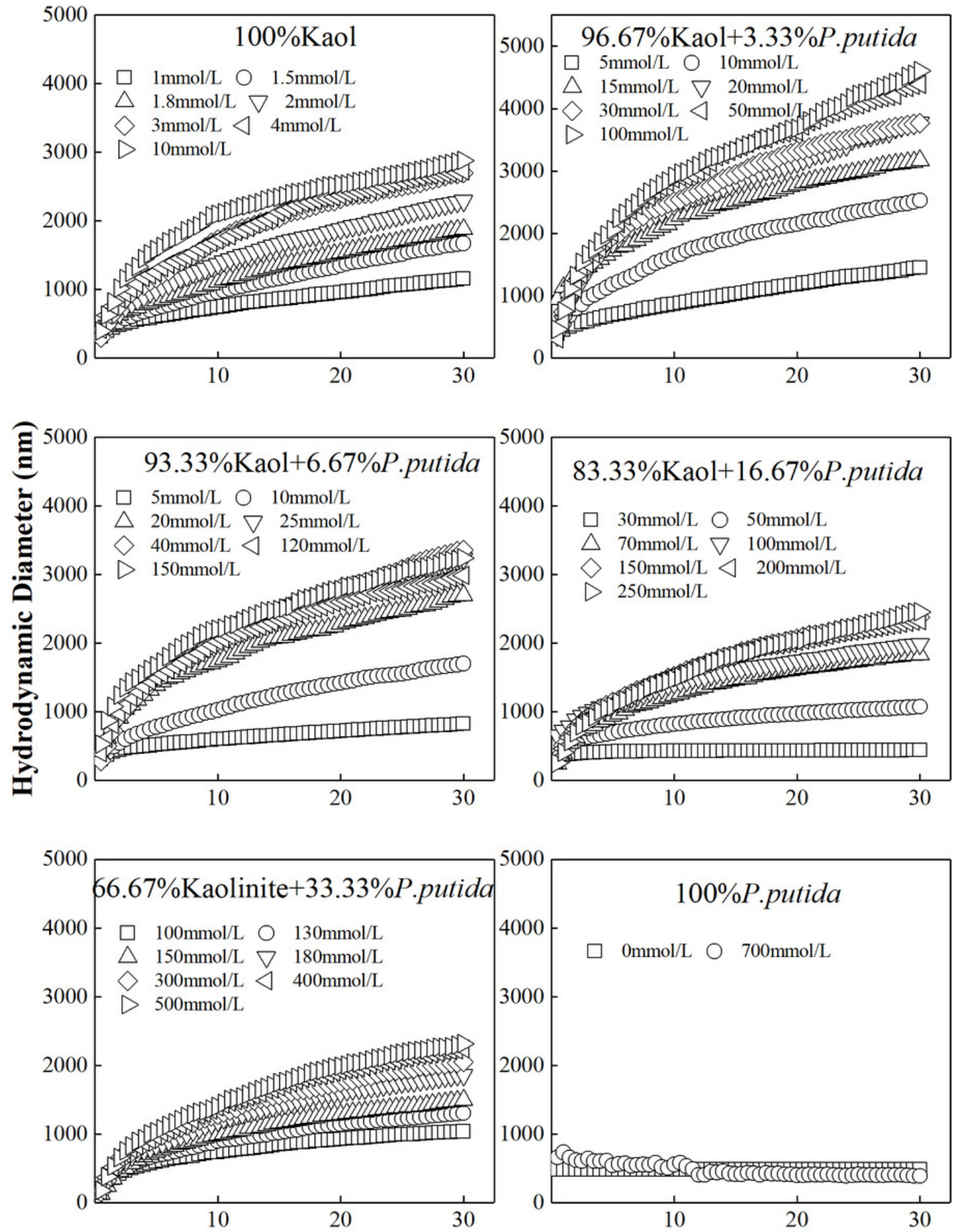

\section{Time (min)}




\section{Figure 3}

Figure. 3 Growth of hydrodynamic diameters of "kaolinite-P. putida" aggregates in $\mathrm{CsNO}_{3}$ solutions. 

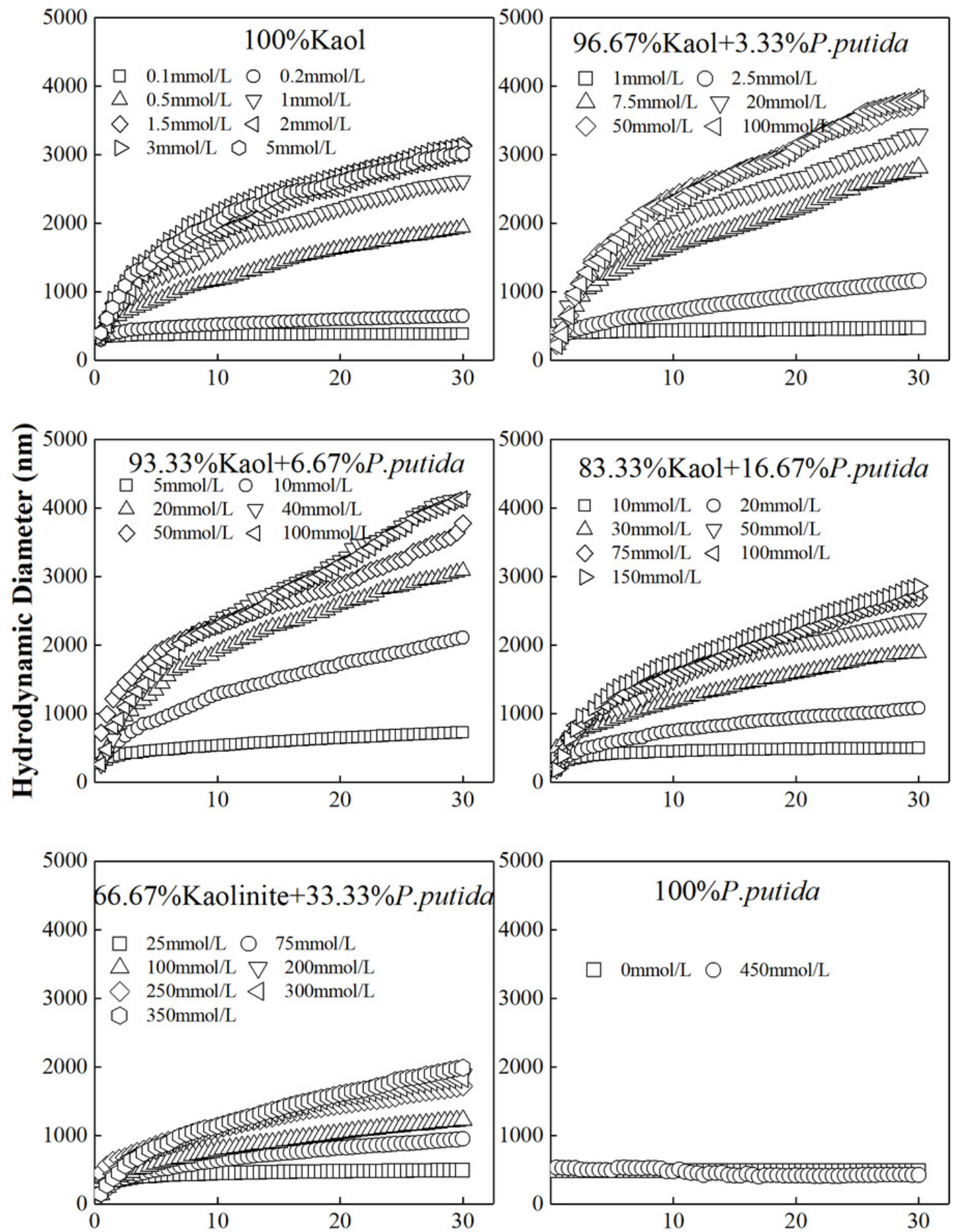

Time (min) 


\section{Figure 4}

Figure 4. The activation energies for the aggregation of the mixture colloids with different bacteria contents in $\mathrm{LiNO}_{3}(\mathrm{~A}), \mathrm{KNO}_{3}(\mathrm{~B})$, and $\mathrm{CsNO}_{3}(\mathrm{C})$ solutions. 

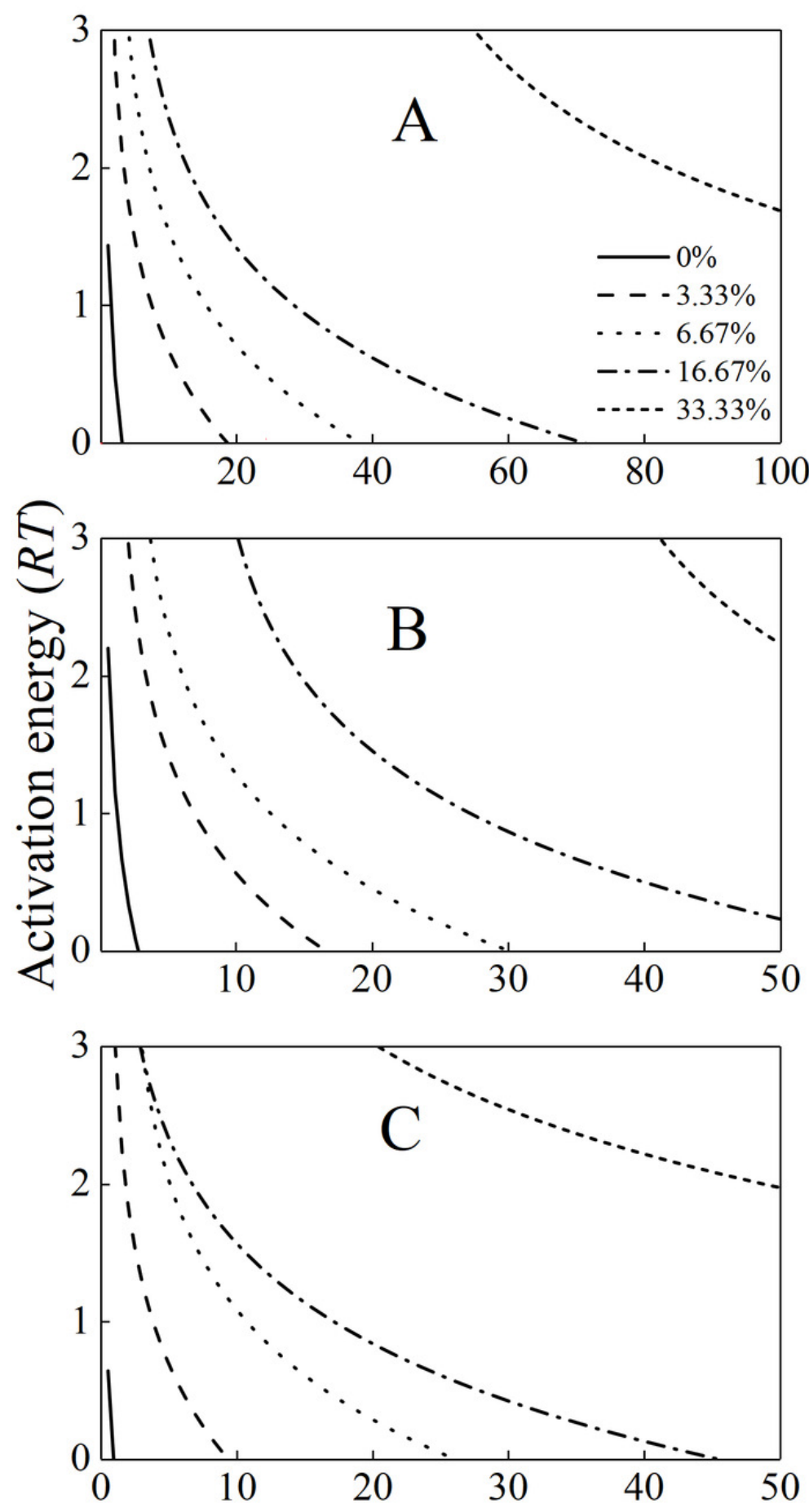

Electrolyte concentration $(\mathrm{mmol} / \mathrm{L})$ 
Figure 5

Figure 5. Zeta potential of "kaolinite-P. putida" mixed particle as a function of $P$. putida content at pH 8.0.

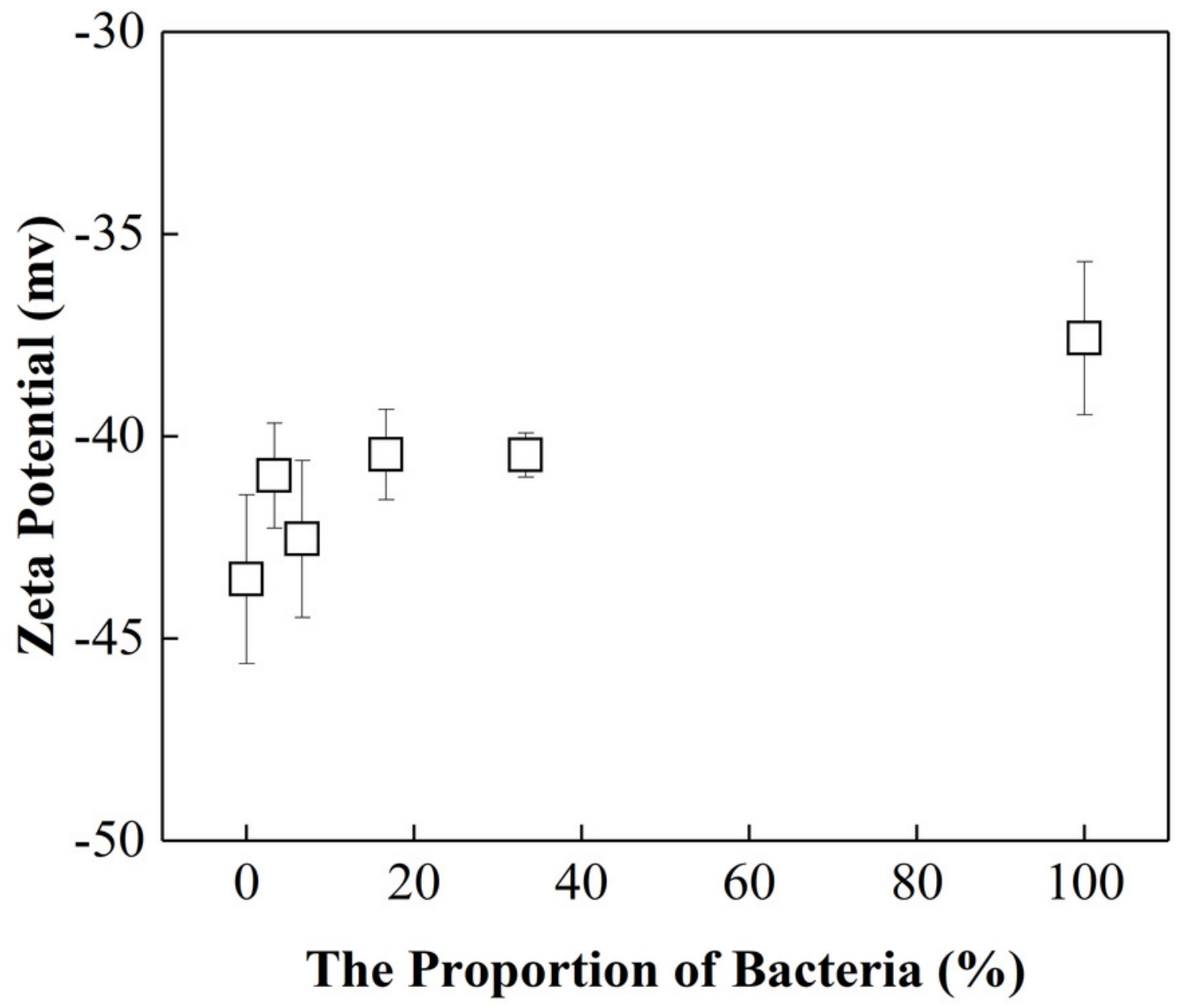


Figure 6

Figure 6. Schematic diagram of "kaolinite-P. putida" TAA rates as a function of electrolyte concentration.

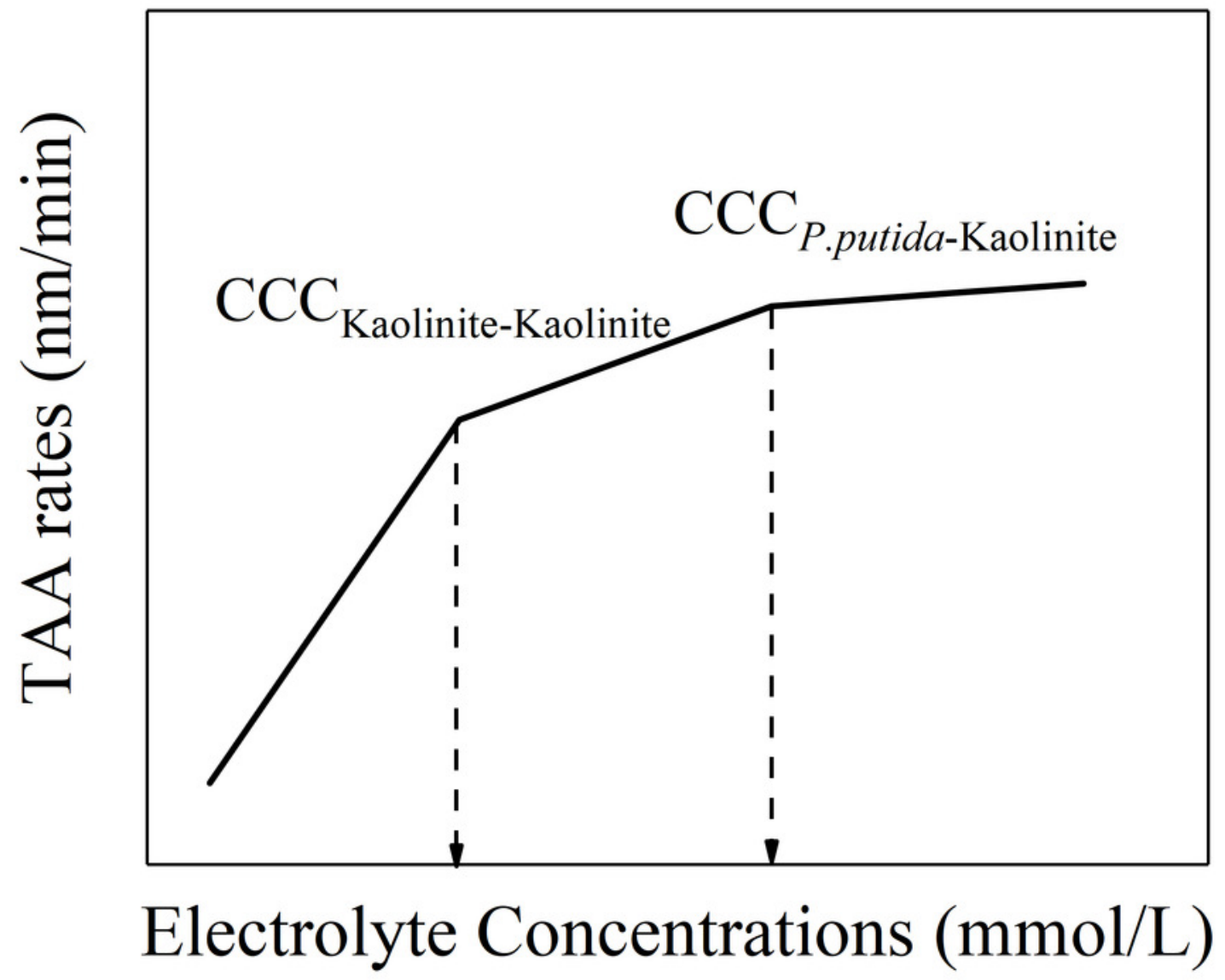




\section{Table $\mathbf{1}$ (on next page)}

Table 1. Expressions of the TAA rates for the aggregation of mixed particles in the various alkali ion solutions when the electrolyte concentration is less than the CCC. 
1 Table 1. Expressions of the TAA rates for the aggregation of mixed particles in the

2 various alkali ion solutions when the electrolyte concentration is less than the CCC.

\begin{tabular}{|c|c|c|c|c|c|c|}
\hline \multirow{2}{*}{$\begin{array}{l}\text { P. putida } \\
\text { contents } \\
(\%)\end{array}$} & \multicolumn{3}{|c|}{$\begin{array}{l}\text { CCC values } \\
(\mathrm{mmol} / \mathrm{L})\end{array}$} & \multicolumn{3}{|c|}{ Expressions of TAA rates vs. electrolyte } \\
\hline & $\mathrm{Li}^{+}$ & $\mathrm{K}^{+}$ & $\mathrm{Cs}^{+}$ & $\mathrm{Li}^{+}$ & $\mathrm{K}^{+}$ & $\mathrm{Cs}^{+}$ \\
\hline 0 & 3.2 & 2.8 & 0.89 & $\begin{array}{l}40.05^{f_{0}} \\
18.52\end{array}$ & $\begin{array}{l}54.64 f_{0} \\
23.68\end{array}$ & $\begin{array}{l}195.09^{f_{0}} \\
16.64\end{array}$ \\
\hline 3.33 & 18.7 & 16.7 & 9.4 & $8.77^{f_{0-8.92}}$ & $\begin{array}{l}13.02 f^{f_{0}} \\
16.92\end{array}$ & $\begin{array}{l}18.24^{f_{0}} \\
10.66\end{array}$ \\
\hline 6.67 & 38.4 & 30.3 & 26.2 & $4.11^{f_{0}-9.56}$ & $\begin{array}{l}5.65^{f_{0}} \\
13.07\end{array}$ & $8.22^{f_{0}}-14.67$ \\
\hline 16.67 & 71.2 & 61.4 & 51.3 & $1.67^{f_{0}-6.12}$ & $\begin{array}{l}1.90^{f_{0}} \\
14.25\end{array}$ & $2.40^{f_{0}}-15.05$ \\
\hline 33.33 & 372.2 & 187.9 & 182.6 & $\begin{array}{l}0.12^{f_{0}} \\
4.697\end{array}$ & $\begin{array}{l}0.54 f_{0} \\
18.23\end{array}$ & $0.40^{f_{0}-5.01}$ \\
\hline
\end{tabular}

